\title{
EFECTO DE MEZCLAS DE FIBRA SEMICOMPOSTEADA CON SUELO EN EL CRECIMIENTO Y LA TOLERANCIA DE ENFERMEDADES EN VIVERO DE PALMA ACEITERA
}

\author{
Gabriel Garbanzo ${ }^{1 / *}$, Eloy Molina* ${ }^{*}$ Edgardo Serrano ${ }^{* *}$, Floria Ramírez.** $^{* * *}$ \\ Palabras clave: Mezcla compost; palma aceitera; nutrición de plantas; enfermedades; vivero. \\ Keywords: Compost mix; oil palm; plant nutrition; diseases; nursery.
}

Recibido: 02/03/17

RESUMEN

Se evaluó el efecto de diferentes mezclas de compost de fibra de racimos vacíos con suelo para sustrato en plantas de palma aceitera en vivero y su efecto sobre la severidad del Complejo de Necrosis Foliar (CNF) en el Pacífico Sur de Costa Rica. Se realizaron mezclas volumétricas de 12,2537 y $50 \%$ de compost de fibra (C) con un Inceptisol eútrico. Se sembraron plantas de palma aceitera del material Compacta x Ghana, se llenaron bolsas de $20 \mathrm{~L}$ y se acomodaron en un diseño experimental de bloques completos al azar, que comprendieron 5 tratamientos y 4 repeticiones. A los 85, 127, 176, 219, 261 y 304 días después de siembra (dds), se evaluó el crecimiento morfológico de las plantas, el porcentaje de severidad de CNF en las hojas y el peso seco de plantas, y se calculó la absorción de nutrimentos. También se evaluó la densidad aparente del suelo (Dap) y la conductividad hidráulica $(\mathrm{CH})$ en las mezclas a los 90 y 300 dds. Se encontró que el tratamiento de $12 \%$ de fibra con suelo mostró significativamente el menor porcentaje de severidad de CNF y presentó el mayor balance porcentual de eficiencia de fertilización de los nutrimentos en comparación con el resto de los tratamientos. La Dap fue significativamente inferior y la $\mathrm{CH}$

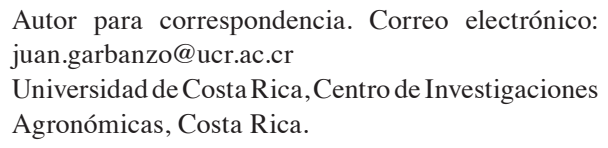
juan.garbanzo@ucr.ac.cr Universidad de Costa Rica, Centro de Investigaciones Agronómicas, Costa Rica.

Aceptado: 01/06/17 
superior en todas las mezclas cuando se comparó con el testigo. Se puede concluir que la mezcla de $12 \%$ de compost con $88 \%$ de suelo mejoró la absorción de nutrimentos en las plantas y aumentó la tolerancia a CNF en las plantas de palma aceitera en vivero.

\section{INTRODUCCIÓN}

La calidad de las plantas en vivero de palma aceitera es fundamental para el establecimiento de sitios de siembra definitivo, debido a que fluye directamente sobre su capacidad de sobrevivir y tolerar ambientes cambiantes según las condiciones agroecológicas (NegrerosCastillo et al. 2010). Sin embargo, para obtener plántulas de calidad se deben mejorar los factores agronómicos, entre estos: fertilización, nutrición, riego, control de problemas fitosanitarios, entre otros. Estos factores influyen directamente con la fisiología y el crecimiento de las plantas, los cuales también se encuentran asociados a condiciones del clima, suelos y ambiente (Fairhurst y Härdter 2012).

El uso de la materia orgánica en los suelos agrícolas tiene amplios beneficios para mejorar las características fisicoquímicas en el tiempo (Ferrera y Alarcón 2001, Cruz-Ruiz et al. 2012). El compost de fibras de fruta fresca de palma aceitera cuando se reincorpora a los sistemas agro-productivos es muy rico en $\mathrm{C}, \mathrm{N}, \mathrm{K}$ y micronutrimentos, ya que aporta parte de la demanda de fertilización del sistema (Miranda y Panduro 2014, Rodríguez et al. 2015), además de reducir la huella de carbono producido en la extracción de la industria. El uso de materia orgánica en los cultivos resulta de vital importancia en relación con la nutrición y manejo de enfermedades. Investigaciones elaboradas en bancos de germoplasma de palma aceitera demostraron que aplicaciones de materia orgánica en la rodaja en conjunto con drenajes y descompactación del suelo, ayuda al crecimiento de las raíces y mejora condiciones control treatment. It can be concluded that $12 \%$ composted fiber with $88 \%$ soil mixture improved nutrient uptake in plants and increased CNF tolerance in nursery oil palm plants.

para prevenir enfermedades, entre estas la Pudrición de Cogollo (Torres 2013, Henry 2015).

En vivero de palma aceitera existen escasos estudios sobre el uso de compost en mezcla con el suelo para mejorar el crecimiento de las plantas. Ensayos realizados con granza de arroz, cascarilla de endocarpo y fibra de mesocarpo de frutas de palma, determinaron que los materiales con menor densidad mostraron mejor facilidad de manipulación al disminuir su peso, no obstante, no encontraron diferencias marcadas con respecto al crecimiento de la raíz y la parte aérea de las palmas entre los sustratos (Umaña et al. 1990). Los mismos autores no cuantifican la absorción de nutrimentos que obtienen las plantas, basándose en fertilizaciones generales de los fertilizantes $18-46-0$ y $15-15-15$.

Investigaciones en el crecimiento de plantas de vivero demostraron un comportamiento estadísticamente similar en la curva de acumulación de materia seca, entre los materiales Deli x LaMé, Deli x Nigeria, Deli x Ghana (Ramírez y Muñoz 2010). Asimismo, en Colombia demostraron que los materiales Tanzania x Ekona, Deli x Yangambi, Deli x Avros y Deli x Yangambi no mostraron diferencias estadísticas en la acumulación de biomasa en el tiempo (Reyes et al. 2008, Reyes et al. 2009). Al realizar el estudio de acumulación de nutrimentos en las plantas, se determinó que el material Deli x LaMé presentó la tendencia de mayor absorción, seguido del material De x Ng y posteriormente de De x Gha, el cual proyectó un consumo de nutrimentos en el siguiente orden decreciente: $\mathrm{N}>\mathrm{K}>\mathrm{Ca}>\mathrm{Mg}>$ P > S (Ramírez y Muñoz 2010). Lo que estableció que las variedades tienen distintos consumos de nutrimentos y que es probable que el aporte 
nutricional que se suministró sea explícitamente de las fertilizaciones y del suelo, ya que ambas investigaciones se realizaron en bolsas con suelo sin presencia de materiales orgánicos.

Por otro lado, en Costa Rica los viveros de palma aceitera han mostrado problemas en el control de enfermedades foliares, principalmente en la zona de Corredores, donde las precipitaciones se encuentran entre 4000 - 6000 $\mathrm{mm} \cdot \mathrm{año} \mathrm{o}^{-1}$. Entre las principales enfermedades foliares reportadas en Corredores, se encuentra la antracnosis (Colletotrichum gloeosporioides) (Ortiz y Fernández 2000, ASD 2010). Sin embargo, las agentes causales reportadas en vivero pueden manifestarse según el estado de desarrollo de la planta, como condiciones climatológicas y manejo. Turner y Bull (1967), Renard y Quillec (1979), Turner (1981) citados por Ulloa y Serrano (2012), reportaron la presencia de otros agentes causales que originan lesiones importantes en las láminas foliares, entre estos se encuentran: Botryodiplodia sp., Melanconium sp., Colletotrichum sp. [Glomerella sp.] y Curvularia sp. Por ser este un problema que involucra a varios patógenos se le denominó Complejo de Necrosis Foliar (CNF) (Ulloa y Serrano 2012). Los efectos reportados según el porcentaje de severidad en las láminas foliares, pueden reducir el crecimiento de las plantas significativamente (Ramírez y Muñoz 2011, Ulloa y Ramírez 2011). Lo cual ocasiona un problema constante en la producción de vivero de palma aceitera.

Debido a las búsquedas de alternativas de producción y manejo de enfermedades en vivero de palma aceitera, esta investigación tuvo el objetivo de evaluar el efecto de mezcla de compost de fibra con el suelo en el crecimiento, los contenidos de nutrimentos y determinar su efecto en la severidad del Complejo de Necrosis Foliar (CNF) en plantas de palma aceitera en fase de vivero.

\section{MATERIALES Y MÉTODOS}

La investigación se llevó cabo en la zona de vivero de la Compañía Palma Tica S.A., ubicada en Coto 47 en el cantón de Corredores en Puntarenas, Costa Rica. Esta zona se encuentra en la llanura aluvial de Coto-Corredores, con una elevación aproximada entre 24 - 30 msnm. La precipitación promedio anual registrada varía entre 3800 - $4500 \mathrm{~mm}$. Se procedió a medir las condiciones climatológicas durante el periodo de evaluación que comprendió desde julio 2014 hasta marzo 2015, se registró una precipitación total $2051 \mathrm{~mm}$. La temperatura promedio durante el periodo fue max: $36,4^{\circ} \mathrm{C}$ y min: $26,5^{\circ} \mathrm{C}$, con una humedad relativa promedio de $87,4 \%$ y una radiación solar promedio de 377,2 Wat.m ${ }^{-2}$.

Se ubicó un sector divido entre drenajes terciarios (boquete) en la zona de vivero con dimensiones de 36 × $23 \mathrm{~m}$ de largo con una zona de topografía plana, cerca al centro del vivero. Se procedió a trasladar suelo (Inceptisol eútrico) en una vagoneta de $12 \mathrm{~m}^{3}$ al boquete dentro del vivero. Los montículos de suelo fueron cubiertos por un plástico trasparente de polietileno durante el tiempo que se procesaba el compost y se recolectó muestras para laboratorio (Cuadro 1).

Cuadro 1. Contenido de nutrimentos en suelo utilizado para las mezclas con compost de fibra de frutos de palma en etapa de vivero.

\begin{tabular}{|c|c|c|c|c|c|c|c|c|c|c|c|c|c|}
\hline \multirow[b]{2}{*}{$\begin{array}{c}\text { ID }^{+} \\
\text {Mehlich } 3\end{array}$} & \multirow[b]{2}{*}{$\begin{array}{c}\mathrm{pH} \\
\mathrm{H}_{2} \mathrm{O}\end{array}$} & \multicolumn{5}{|c|}{$\mathrm{cmol}(+) \cdot .^{-1}$} & \multirow[b]{2}{*}{$\begin{array}{c}\% \\
\text { SA }\end{array}$} & \multicolumn{6}{|c|}{$\mathrm{mg} . \mathrm{l}^{-1}$} \\
\hline & & $\begin{array}{c}\text { Acidez } \\
(\mathrm{KCl})\end{array}$ & $\mathrm{Ca}$ & $\mathrm{Mg}$ & K & CICE & & $\mathrm{P}$ & $\mathrm{Zn}$ & $\mathrm{Cu}$ & $\mathrm{Fe}$ & $\mathrm{Mn}$ & $\mathrm{Si}^{*}$ \\
\hline $\mathrm{NC}$ & 5,5 & 0,5 & 4 & 1,1 & 0,2 & 5 & & 15 & 1,7 & 0,4 & 10 & 4 & 200 \\
\hline Suelo inicio & 6,24 & 0,28 & 35,2 & 3,97 & 0,46 & 39,9 & 0,70 & 18,2 & 2,52 & 12,3 & 283 & 57 & 173 \\
\hline
\end{tabular}

+Solución extractora Mehlich 3, laboratorio suelos y foliares Palma Tica S.A.; NC = nivel crítico para Inceptisoles según Cabalceta y Molina (2006). CICE $=$ Capacidad de intercambio de Cationes Efectiva $=$ Acidez $+\mathrm{Ca}+\mathrm{Mg}+\mathrm{K} . *$ Metodología de extracción con ácido acético según Lobo (2013). 
Se procedió a compostear fibra de estopa o racimos vacíos de palma aceitera durante 30 días en un invernadero, removiendo los residuos diariamente, junto con aplicaciones de efluente residual de la planta extractora. A los 25 días de compostaje se procedió a realizar 5 análisis químicos en distintos puntos de la cama, con el fin de determinar su concentración de nutrimentos totales (Cuadro 2), se dejó por 5 días en el invernadero y después se trasladó $12 \mathrm{~m}^{3}$ de compost de fibra al vivero, se cubrió con un plástico de polietileno transparente por una semana y se realizó un sexto análisis químico total del compost.

Cuadro 2. Determinación de nutrimentos totales a los 25 días de compostaje en fibra de palma aceitera en invernadero y vivero.

\begin{tabular}{lc|ccccccc}
\hline \multirow{2}{*}{ Nutrimento } & & Fibra M1 & Fibra M2 & Fibra M3 & Fibra M4 & Fibra M5 & $\begin{array}{c}\text { F. Vivero } \\
\text { M6 }{ }^{*}\end{array}$ & $\bar{X}$ \\
\hline $\mathrm{pH}$ & $\mathrm{H}_{2} \mathrm{O}$ & 9,04 & 9,17 & 9,24 & 9,21 & 9,05 & 8,54 & 9,04 \\
\hline $\mathrm{N}$ & & 2,22 & 1,81 & 1,96 & 1,76 & 2,07 & 1,69 & 1,92 \\
$\mathrm{C}$ & 40,6 & 41,7 & 40,9 & 41,4 & 41,4 & 42,7 & 41,5 \\
$\mathrm{C} / \mathrm{N}$ & 18,3 & 23,1 & 20,9 & 23,5 & 20,0 & 25,3 & 21,8 \\
$\mathrm{P}$ & & 0,48 & 0,41 & 0,45 & 0,35 & 0,4 & 0,39 & 0,41 \\
$\mathrm{~K}$ & \multirow{3}{*}{$*$} & 4,02 & 3,54 & 3,78 & 3,70 & 3,70 & 3,14 & 3,65 \\
$\mathrm{Ca}$ & 1,26 & 0,98 & 1,11 & 0,88 & 1,04 & 1,01 & 1,05 \\
$\mathrm{Mg}$ & & 0,73 & 0,6 & 0,68 & 0,55 & 0,61 & 0,62 & 0,63 \\
$\mathrm{~S}$ & & 0,34 & 0,28 & 0,29 & 0,27 & 0,31 & 0,31 & 0,30 \\
$\mathrm{Si}$ & & - & - & - & - & - & 0,85 & - \\
\hline $\mathrm{Fe}$ & & 2089 & 1728 & 1873 & 1508 & 1811 & 1985 & 1832 \\
$\mathrm{Cu}$ & & 46,4 & 38,1 & 43,4 & 38,7 & 41,2 & 41,5 & 41,6 \\
$\mathrm{Zn}$ & $\mathrm{mg} \cdot \mathrm{kg}^{-1}$ & 69 & 57 & 71 & 54 & 64 & 59 & 62,3 \\
$\mathrm{Mn}$ & & 122 & 102 & 118 & 88 & 104 & 107 & 106,8 \\
$\mathrm{~B}$ & & 33 & 28 & 31 & 27 & 30 & 25 & 29,0 \\
\hline $\mathrm{Humedad}$ & $\%$ & 70,5 & 69,2 & 69,5 & 67,8 & 69,1 & 67,2 & 68,9 \\
\hline
\end{tabular}

Fibra con una semana de estar bajo cubierta en área de vivero; pH: relación material: agua 1:16.7. AOAC 973.04. Elementos: extracción por microondas. Detección ICP-OES Óptima 8500. C/N por combustión seca. CHN.

Según investigaciones preliminares con mezclas de materia orgánica y suelo en los viveros comerciales de Palma Tica S.A, se diseñaron 4 mezclas potenciales para evaluar, a partir de un $50 \%$ de cada material (Cuadro 3). Posteriormente, se cubicó un "balde" de montacargas de $0,5 \mathrm{~m}^{3}$ para la estimación de diluciones de compost con suelo. Asimismo, la elaboración de los tratamientos se realizaron mediante dilución de las mezclas madres; estas se efectuaron de la siguiente manera: se mezcló 12 baldes de suelo y 12 baldes de compost en un montículo sobre el área a llenar las bolsas, estas se removieron constantemente hasta que los materiales fueran homogéneos, de esta forma, se realizó la mezcla 50\% suelo y 50\% compost (mezcla 1). Posteriormente, se llenaron 9 baldes de la mezcla 1 y 9 baldes de suelo, se removieron simultáneamente hasta lograr una homogenización de los materiales y concluir con las proporción de $75 \%$ suelo y $25 \%$ compost (mezcla 2). Continuamente, se combinaron 5 baldes de la mezcla 1 y 5 baldes de la mezcla 2 , se homogenizaron para definir con la mezcla $62,5 \%$ suelo y $37 \%$ compost. Por último, se diluyeron 4 baldes de la mezcla 2 con 4 baldes de suelo para lograr la mezcla $87,5 \%$ suelo y $12,5 \%$ compost. 
Cuadro 3. Tratamientos de mezclas de compost y suelos.

\begin{tabular}{lll}
\hline Mezcla & Id. & \multicolumn{1}{c}{ Tratamientos } \\
\hline 1 & $50 \mathrm{C}$ & $50 \%$ Suelo $+50 \%$ Compost \\
2 & $25 \mathrm{C}$ & $75 \%$ Suelo $+25 \%$ Compost \\
3 & $37 \mathrm{C}$ & $62,5 \%$ Suelo $+37,5 \%$ Compost \\
4 & $12 \mathrm{C}$ & $87,5 \%$ Suelo $+12,5 \%$ Compost \\
5 & $\mathrm{~T}$ & $100 \%$ Suelo \\
\hline
\end{tabular}

Posteriormente, se procedió a cubrir las mezclas con plástico transparente de polietileno durante una semana. Luego se llenaron las bolsas de vivero con las distintas mezclas, se removió nuevamente el montículo con tridentes para separar las estopas cementadas con el suelo y se mezcló nuevamente. Asimismo, se llenaron las bolsas de $20 \mathrm{~L}$ con las distintas mezclas de compost y suelo, se efectuaron golpes leves para comprimir y eliminar las cavidades de aire, se alinearon de acuerdo con el mapa experimental y se etiquetaron con números para sus respectivas evaluaciones.

Se seleccionaron plantas de E. guineensis del material Compacta x Ghana. Estas fueron sembradas en pre-vivero con turba acondicionada marca "Jiffi" y fueron manejadas durante 2 meses bajo condiciones controladas. La nutrición de estas plántulas se manejó bajo fuentes de lenta liberación (Osmocote ${ }^{\circledR}$ ) y aplicaciones foliares en conjunto con fungicidas preventivos. Las plantas se seleccionaron de manera que el material fuera totalmente homogéneo, se desecharon plantas alongadas, con presencia de enfermedades foliares, mal desarrolladas, entre otros.

Previo a la siembra de las plantas, se realizó una aplicación de herbicida en toda el área de vivero con el fin de realizar un manejo total de las malezas. Dos semanas después se trasplantaron plántulas de E. guineensis. Se realizó el mismo manejo agronómico utilizado por el departamento de viveros, con excepción de la nutrición y el control de enfermedades foliares. La fórmula que se utilizó en vivero corresponde a 15,50 - 9,76 $15,50-4,00-3,20(\mathrm{~S})-0,30(\mathrm{~B})$; las cuales están constituidas por las fuentes de nitrato de amonio (NAM), Microessentials (SZ), cloruro de potasio $(\mathrm{KCl})$, kiesierita y granubor. Esta fórmula se calculó según los requisitos calculados mediante la curva de absorción propuesta por Ramírez y Muñoz (2010) (Cuadro 4). La fertilización se aplicó cada 15 días, esta fue de forma granular en el área superficial de la bolsa, distanciados del bulbo para no producir pérdida de agua en la planta por difusión.

Cuadro 4. Fertilización propuesta con base en la curva de absorción en plantas de E. guinensis en etapa de vivero.

\begin{tabular}{lccccccc}
\hline Consumo curva & $\mathrm{N}$ & $\mathrm{P}$ & $\mathrm{K}$ & $\mathrm{Mg}$ & $\mathrm{S}$ & $\mathrm{Zn}$ & $\mathrm{B}$ \\
Eficiencia*/dds** & $60 \%$ & $50 \%$ & $70 \%$ & $80 \%$ & $50 \%$ & $50 \%$ & $10 \%$ \\
\hline & & & & --------- g.planta ${ }^{-1}----------$ & & \\
82 & 0,05 & 0,01 & 0,03 & 0,02 & 0,01 & 0,15 & 0,63 \\
149 & 0,93 & 0,09 & 0,26 & 0,07 & 0,06 & 1,01 & 2,03 \\
210 & 2,79 & 0,27 & 1,03 & 0,24 & 0,19 & 2,53 & 6,52 \\
268 & 5,94 & 0,72 & 2,98 & 0,56 & 0,51 & 8,29 & 25,02 \\
298 & 8,71 & 1,03 & 4,76 & 0,80 & 0,73 & 11,52 & 29,91 \\
335 & 17,48 & 2,25 & 10,28 & 1,69 & 1,64 & 29,13 & 61,77 \\
\hline
\end{tabular}

*Eficiencia fertilización propuesta. **días después de siembra. 


\section{Variables evaluadas}

Se realizaron muestreos a los $85,127,176$, 219, 261, 304 días después de siembra (dds), para determinar el crecimiento de la planta. Se midieron con una cinta métrica el largo de raquis que comprende el tamaño desde la parte proximal de peciolo hasta el meristemo apical de la hoja. El número de hojas totales en las plantas y con un calibre de vernier se midió el diámetro de bulbo que se encuentra a nivel del suelo.

Las evaluaciones de severidad del complejo de necrosis foliar (CNF) se realizaron mediante una escala visual propuesta por la Compañía Palma Tica S.A. La escala está representada por 5 grados que determinan el porcentaje de daño en la hoja 1 y toda la planta. Están constituidos de la siguiente manera: $1=$ representa el 3\% de la hoja o planta dañada, $2=$ $7 \%, 3=15 \%, 4=40 \%, 5=75 \%$. Se realizaron evaluaciones de severidad en la hoja 1 y planta total. Se digitaron los datos de crecimiento para estimar una planta promedio para el análisis de absorción de acuerdo con las mediciones.

La selección de plantas para el análisis de absorción en vivero se realizó por medio de una media estimada a partir de los datos morfológicos. Cada repetición estaba compuesta por 28 - 24 plantas donde se calculó un intervalo de confianza para el diámetro de bulbo, largo de raquis y hojas totales. El intervalo de confianza se calculó a partir de la desviación estándar, el tamaño de la muestra con un alfa $(\alpha)=0,01$. Posteriormente, se determinó el límite superior y el límite inferior según la media de los datos. La selección de las plantas para análisis de absorción se estimó mediante un filtrado de los datos para cada repetición, donde se incluían los límites inferiores y superiores correspondientes a cada una de las variables morfológicas. En las repeticiones que mostraban más de 2 plantas con los intervalos de confianza estimados, se seleccionaba la planta que mostraba el diámetro de bulbo más cercano a la media.

Se recolectó una planta por repetición a los 85, 127, 176, 219, 261, 304 dds; se lavó el adobe (mezcla) con abundante agua en presencia de un sarán, para evitar la pérdida de raíces, y posteriormente, se pesaron la parte aérea y las raíces. Ambas partes se secaron en un horno durante 3 días a $60^{\circ} \mathrm{C}$ y se procedió a pesar el peso seco de las plantas. Después, se enviaron al laboratorio para el respectivo análisis de nutrimentos de la parte área y raíz.

Por otro lado, se realizó un muestreo foliar según la metodología de Rodríguez y Jiménez (2013), el cual consistió en cortar la hoja 1 para el caso de las hojas bifurcadas y lanceoladas, para el caso de hojas compuestas totalmente abiertas se recolectaron 2 folios a cada lado de la parte media, se extrajo las venas de las hojas y se secaron a $60^{\circ} \mathrm{C}$ durante 3 días. En ambas muestras se realizaron determinaciones de $\mathrm{Si}$, mediante la metodología de Lobo (2013), N por combustión seca, $\mathrm{P}, \mathrm{K}, \mathrm{Ca}, \mathrm{Mg}, \mathrm{S}, \mathrm{Fe}, \mathrm{Zn}, \mathrm{Mn}, \mathrm{Cu}$, a través de la digestión húmeda con $\mathrm{HNO}_{3}$ y determinación por espectrometría de emisión atómica con plasma (ICP).

Los análisis químicos en suelos se realizaron a los 90, 170, 260 dds. Las muestras se recolectaron a 2 profundidades de $0-15$ y $15-$ 30 por medio de un submuestreo en 15 bolsas por repetición alternadas entre plantas. Las muestras fueron enviadas al Laboratorio de Suelos y Foliares de la Compañía Palma Tica S.A., se determinaron $\mathrm{pH}$, Acidez Intercambiable, $\mathrm{Ca}, \mathrm{Mg}, \mathrm{K}, \mathrm{P}$, $\mathrm{Fe}, \mathrm{Zn}, \mathrm{Mn}, \mathrm{Cu}, \mathrm{Si}$ y Al mediante extracciones con Mehlich III de acuerdo con la metodología de Cabalceta y Molina (2006) y las determinaciones de Si por el método de Lobo (2013).

Las variables físicas se evaluaron en las mezclas de compost de fibra una vez sembradas las plantas en las bolsas. Estas fueron la densidad aparente y conductividad hidráulica, las cuales se determinaron mediante el método de Forsythe (1980). Las muestras fueron tomadas a los 90 y $320 \mathrm{dds}$, estas se obtuvieron de los primeros $10 \mathrm{~cm}$ de la superficie de suelo en la bolsa, por medio de cilindros de acero inoxidable insertados bajo presión. Posteriormente, fueron extraídos con una espátula y envueltos en papel aluminio para luego enviarlos al Laboratorio de Física de 
Suelos del Departamento de Investigación de Palma Tica S.A.

La infiltración de agua en los suelos de las bolsas se realizó mediante la metodología de calidad de suelos USDA (2001). Esta metodología consistió en insertar un cilindro de 6 pulgadas de diámetro en el suelo y cuantificar el tiempo que tarda en infiltrar una pulgada de agua. Sin embargo, esta metodología se adaptó para las bolsas de vivero, con un cilindro de 2 pulgadas de diámetro, lo cual permitió evaluar la infiltración en el área de la superficie de las bolsas.

\section{Diseño experimental}

El experimento fue constituido por 5 tratamientos y 4 repeticiones con un diseño experimental de bloques completos al azar. Los bloques fueron ubicados en un sector céntrico, con historial de enfermedades foliares y sin problemas de topografía. Se prepararon 4 bloques, cada bloque fue representado por 210 plantas con un tamaño de $265 \mathrm{~m}^{2}$, cada repetición tenía un total de 30 plantas.

Para el análisis de los datos morfológicos se realizaron índices de áreas bajo la curva (abc), para reunir la información de las variables en el tiempo (Ecuación 1). Esta metodología consistió en calcular el área de un trapecio entre 2 fechas de evaluación; se consideraron las evaluaciones morfológicas en cada fecha y los días transcurridos para realizar una sumatoria de las áreas calculadas entre los periodos. La sumatoria de las áreas determinó el índice de abc, lo cual mide el efecto de los tratamientos en trascurso del tiempo.

$$
\sum \frac{b+B \times a}{2}
$$

Ecuación 1
Donde:

$\mathrm{B}=$ medida en el tiempo 2 (t1)

$\mathrm{b}=$ medida en el tiempo 1 (t2)

$\mathrm{a}=$ número de días trascurridos entre $\mathrm{t} 1$ y $\mathrm{t} 2$

Seguidamente, los datos fueron sometidos a un análisis de varianza, se calcularon las diferencias estadísticas a través de pruebas de Dunnett test $(\alpha=0,05)$ para los análisis morfológicos y LSD $(\alpha=0,05)$ para los análisis químicos. Por último, para explicar la variabilidad del sistema, se procedió a realizar un análisis multivariado mediante un análisis de componentes principales (CP) (Abdi y Williams 2010, Jolliffe 2005, Serrano 2003). Con el fin de encontrar una posible estructura en la base de datos y explicar la variación según las dimensiones calculadas. Los cálculos fueron realizados con el programa estadístico Rstudio versión 0.98.1102.

\section{RESULTADOS}

\section{Efecto de las mezclas con compost en el crecimiento de plantas y la severidad de CNF}

La aplicación de compost aumentó el crecimiento y disminuyó la severidad del CNF en vivero de palma aceitera (Cuadro 5). Al evaluar las variables morfológicas de crecimiento y severidad en vivero, se encontró que el diámetro de bulbo según el índice de área bajo la curva (abc), no mostró diferencias estadísticas significativas $(\alpha=0,05)$ cuando se compararon los tratamientos con el testigo $(631,1)$. Sin embargo, se encontraron tendencias de mayor diámetro de bulbo con el procedimiento $12 \mathrm{C}(646,1)$. El abc calculado para el largo de hoja 1 no mostró diferencias estadísticas al comparar el testigo con el resto de las pruebas. 
Cuadro 5. Índice de área bajo la curva en crecimiento morfológico y severidad de CNF en plantas de palma aceitera, tratadas con distintas mezclas de compost de fibra y suelo en fase de vivero.

\begin{tabular}{|c|c|c|c|c|c|c|}
\hline \multirow[t]{2}{*}{ Tratamientos } & $\begin{array}{c}\text { Diámetro } \\
\text { bulbo }\end{array}$ & $\begin{array}{l}\text { Largo } \\
\text { Hoja }\end{array}$ & $\begin{array}{l}\text { Largo } \\
\text { raquis }\end{array}$ & $\begin{array}{l}\text { Hojas } \\
\text { totales }\end{array}$ & $\begin{array}{c}\text { Severidad } \\
\text { Hoja } 1\end{array}$ & $\begin{array}{c}\text { Severidad } \\
\text { Total }\end{array}$ \\
\hline & \multicolumn{6}{|c|}{ 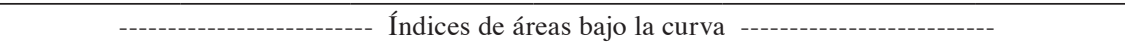 } \\
\hline $12 \mathrm{C}$ & 646,1 & 733,1 & 3107,2 & 3107,2 & 1062,4 & 1782,1 \\
\hline $25 \mathrm{C}$ & 638,5 & 727,2 & 2986,5 & 2986,5 & 1106,0 & 1857,1 \\
\hline $37 \mathrm{C}$ & 625,2 & 736,4 & 3034,4 & 3034,4 & 1134,0 & 1815,9 \\
\hline $50 \mathrm{C}$ & 617,6 & 706,2 & 2861,5 & 2861,4 & 1083,7 & 1949,5 \\
\hline Testigo & 631,1 & 763,5 & 3210,9 & 3210,9 & 1175,8 & 2019,5 \\
\hline Comparaciones & \multicolumn{6}{|c|}{ - Dunnett test $\operatorname{Pr}(>|t|)$ - } \\
\hline $12 \mathrm{C}-$ Testigo & 0,51 & 0,16 & 0,49 & 0,53 & 0,53 & 0,03 \\
\hline $25 \mathrm{C}-$ Testigo & 0,92 & 0,07 & 0,02 & 0,11 & 0,68 & 0,18 \\
\hline $37 \mathrm{C}-$ Testigo & 0,97 & 0,25 & 0,09 & 0,05 & 0,85 & 0,10 \\
\hline $50 \mathrm{C}-$ Testigo & 0,60 & 0,001 & $<0,001$ & 0,99 & 0,65 & 0,65 \\
\hline
\end{tabular}

$\mathrm{n}=18801$; Variables morfológicas y severidad evaluados a los 85, 127, 176, 219, 261, 304 días después de siembra. Probabilidades por debajo de $5 \%(\operatorname{Pr}(>|t|)<0,05)$ representan diferencias significativas de tratamientos en comparación al testigo.

El largo de raquis mostró el menor crecimiento reportado en 25C $(2986,5)$ y $50 \mathrm{C}(2861,5)$ cuando se comparó con el testigo $(3210,9)$. Al evaluar el número de hojas se encontró que el tratamiento 37C mostró el menor número de hojas $(3034,4)$ cuando se comparó con el testigo $(3210,9)$. El abc calculado en la severidad de la hoja 1 no mostró diferencias estadísticas entre las pruebas al compararlo con el testigo, sin embargo, existe tendencia de menor severidad de CNF respecto al de $12 \mathrm{C}$. La severidad de CNF en las hojas totales de la planta mostró que ese último (12C) presentó la menor severidad $(1782,1)$ con diferencias significativas cuando se comparó con el testigo $(2019,5)$.
Las mezclas de compost de fibra de palma con el suelo mostraron tendencias de mayor acumulación de biomasa seca en las plantas de vivero (Cuadro 6). Al comparar el abc de peso húmedo y seco aéreo con el testigo, no se encontraron diferencias estadísticas significativas, tampoco el abc de peso húmedo y seco de raíz cuando se compararon los tratamientos con el testigo, lo mismo se identificó al realizar la sumatoria de los pesos secos de raíz y parte aérea donde se calculó el abc de peso seco total respecto al testigo correspondiente. Sin embargo, en todos los pesos tanto aéreo, raíz y total se encontró tendencias de mayor acumulación de biomasa en el tiempo con el tratamiento $12 \mathrm{C}$ cuando se compararon el resto de las pruebas incluyendo el testigo. 
Cuadro 6. Índice de área bajo la curva en peso húmedo, seco y peso seco total en plantas de palma aceitera, tratadas con distintas mezclas de compost de fibra con suelo en fase de vivero.

\begin{tabular}{|c|c|c|c|c|c|}
\hline \multirow{2}{*}{ Tratamientos } & Peso húmedo aérea & Peso seco aérea & Peso húmedo Raíz & Peso seco Raíz & Peso seco total \\
\hline & \multicolumn{5}{|c|}{ - } \\
\hline $12 \mathrm{C}$ & 34455,7 & 8719,9 & 11127,6 & 1805,2 & 10525,1 \\
\hline $25 \mathrm{C}$ & 30885,8 & 7709,1 & 10761,3 & 1715,3 & 9424,3 \\
\hline $37 \mathrm{C}$ & 30833,7 & 7539,1 & 10583,2 & 1615,1 & 9154,2 \\
\hline $50 \mathrm{C}$ & 31489,6 & 6855,1 & 11043,6 & 1704,2 & 8559,3 \\
\hline Testigo & 31190,1 & 7646,8 & 12392,6 & 1767,7 & 9414,5 \\
\hline Comparaciones & \multicolumn{5}{|c|}{ - Dunnett test $\operatorname{Pr}(>|t|)$} \\
\hline $12 \mathrm{C}-\mathrm{Test}$ & 0,70 & 0,28 & 0,80 & 1,00 & 0,45 \\
\hline $25 \mathrm{C}-\mathrm{Test}$ & 1,00 & 1,00 & 0,64 & 1,00 & 1,00 \\
\hline $37 \mathrm{C}$ - Test & 1,00 & 0,99 & 0,56 & 0,92 & 1,00 \\
\hline $50 \mathrm{C}-$ Test & 1,00 & 0,52 & 0,77 & 1,00 & 0,67 \\
\hline
\end{tabular}

$\mathrm{n}=120$; Variables de crecimiento evaluadas a los 85, 132, 180,223, 265, 308 días después de siembra; Probabilidades por debajo de $5 \%(\operatorname{Pr}(>|\mathrm{t}|)<0,05)$ representan diferencias significativas de tratamientos en comparación al testigo.

Los resultados con compost de fibra de palma mostraron un mayor crecimiento en las plantas cuando se evaluaron a los 300 dds (Cuadro 7). Al evaluar las variables morfológicas y severidad de CNF en la planta a los 300 dds, el diámetro de bulbo $(\mathrm{cm})$ mostró diferencias altamente significativas $(\alpha<0,001)$ cuando se comparó el tratamiento $12 \mathrm{C}(5,88 \mathrm{~cm})$ contra el testigo $(5,40 \mathrm{~cm})$. En relación con el largo de raquis se encontró un menor crecimiento en el de $50 \mathrm{C}(35,24 \mathrm{~cm})$ y mostró diferencias estadísticas $(\alpha<0,001)$ cuando se comparó contra el testigo $(38,96 \mathrm{~cm})$. El número de hojas totales y el peso seco total en las plantas no mostraron diferencias

Cuadro 7. Crecimientos morfológicos, peso seco total y severidad de plantas de palma aceitera a los 300 dds, tratadas con distintas mezclas de compost de fibra con suelo en fase de vivero.

\begin{tabular}{|c|c|c|c|c|c|c|}
\hline Tratamientos & $\begin{array}{l}\text { Diámetro } \\
\text { bulbo }\end{array}$ & Largo raquis & $\begin{array}{l}\text { Hojas } \\
\text { totales }\end{array}$ & $\begin{array}{c}\text { Peso Seco } \\
\text { Total }\end{array}$ & $\begin{array}{l}\text { Severidad } \\
\text { Hoja } 1\end{array}$ & $\begin{array}{c}\text { Severidad } \\
\text { Total }\end{array}$ \\
\hline & $\mathrm{cm}$ & $\mathrm{cm}$ & $\mathrm{u}$ & $g$ & $\%$ & $\%$ \\
\hline $12 \mathrm{C}$ & 5,88 & 37,81 & 9,45 & 161,03 & $4,71 \mathrm{a}$ & $8,58 \mathrm{a}$ \\
\hline $25 \mathrm{C}$ & 5,52 & 36,79 & 9,39 & 151,28 & $3,46 \mathrm{a}$ & $8,36 \mathrm{a}$ \\
\hline $37 \mathrm{C}$ & 5,62 & 36,96 & 9,42 & 129,13 & $4,28 \mathrm{a}$ & 8,74 a \\
\hline $50 \mathrm{C}$ & 5,46 & 35,24 & 9,19 & 134,41 & $4,16 \mathrm{a}$ & $8,44 \mathrm{a}$ \\
\hline Testigo & 5,40 & 38,96 & 9,21 & 141,53 & $4,79 \mathrm{a}$ & $9,42 \mathrm{a}$ \\
\hline Comparaciones & \multicolumn{4}{|c|}{ Dunnett test $\operatorname{Pr}(>|t|)$} & \multicolumn{2}{|c|}{ Kruskal Wallis } \\
\hline $12 \mathrm{C}-$ Testigo & $<0,001$ & 0,73 & 0,60 & 0,51 & - & - \\
\hline $25 \mathrm{C}-$ Testigo & 0,68 & 0,21 & 0,82 & 0,91 & - & - \\
\hline $37 \mathrm{C}-$ Testigo & 0,18 & 0,24 & 0,71 & 0,82 & - & - \\
\hline $50 \mathrm{C}-$ Testigo & 0,96 & 0,003 & 1,00 & 0,97 & - & - \\
\hline
\end{tabular}

$\mathrm{n}=2476$; Variables de crecimiento evaluadas a los 85, 132, 180, 223, 265, 308 días después de siembra; Probabilidades por debajo de $5 \%(\alpha<0,05)$ representan diferencias significativas de tratamientos en comparación al testigo. 
significativas cuando se compararon con el testigo, sin embargo, el experimento $12 \mathrm{C}$ mostró tendencias de mayor número de hojas $(9,45)$ y peso seco (161 g) que los demás ensayos. La severidad del CNF en la hoja 1 y total en la planta no mostró diferencias al compararlos a los 300 dds, no obstante, los resultados mostraron tendencias de menor \% de severidad al compararlo con el modelo referente.

\section{Efecto de las mezclas con compost en la concentración de nutrimentos en el suelo}

El pH del suelo disminuyó y la acidez y el aluminio intercambiable aumentaron con la edad de las plantas en vivero (Cuadro 8). Al comparar el $\mathrm{pH}$ en los primeros $15 \mathrm{~cm}$ de profundidad, se encontró una disminución significativa entre los 85 dds y 265 dds, donde 50C y $12 \mathrm{C}$ mostraron el mayor $\mathrm{pH}(5,54$ y 5,22$)$ en el último muestreo.
Al comparar el $\mathrm{pH}$ a una profundidad de $15-30$ cm se encontró el mismo desempeño, sin embargo, al comparar las 2 profundidades se encontró mayor $\mathrm{pH}$, a mayor profundidad. La acidez intercambiable en los primeros $15 \mathrm{~cm}$ de profundidad mostró un incremento significativo $(\alpha=0,05)$ entre los 85 y 265 dds, y se identificó que $25 \mathrm{C}$ y el testigo, mostraron mayor acidez en comparación al resto al ser superior al nivel crítico. Al evaluar la acidez a una profundidad de $15-30 \mathrm{~cm}$ se identificó que no superó el nivel crítico y se mantuvo constante en el tiempo, a excepción del testigo, el cual reveló rangos superiores al nivel crítico a los 175 y 265 dds. El Al intercambiable en ambas profundidades no presentó incrementos importantes en las 2 primeras fechas de evaluación, sin embargo, a los 265 dds el testigo mostró la mayor concentración de Al significativamente al compararlo con el resto de las pruebas. 


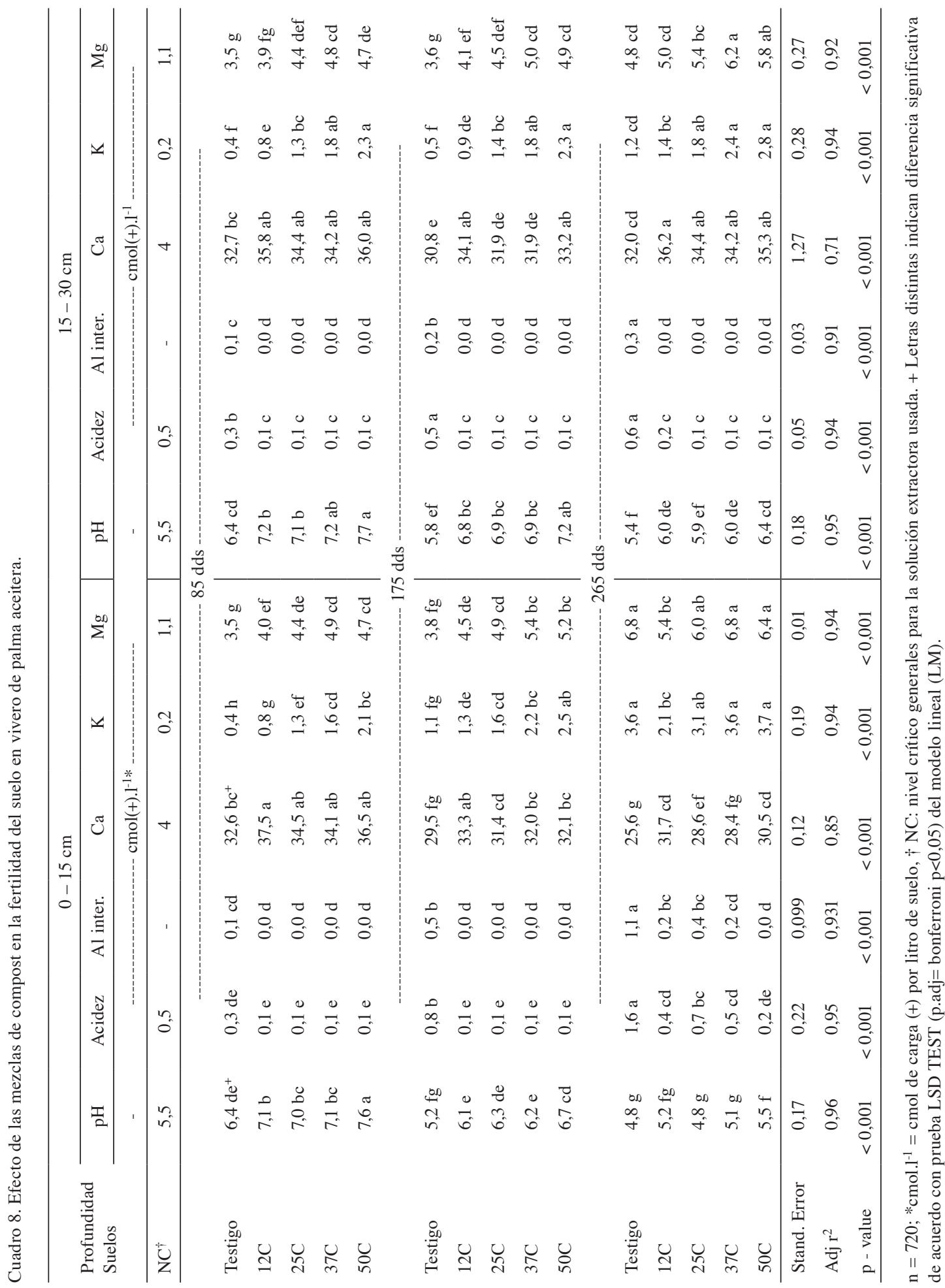


La concentración de las bases intercambiables evaluadas en los suelos mezclados con compost de fibra, mostraron resultados sobre el nivel crítico, un decrecimiento en el $\mathrm{Ca}$ y una acumulación de $\mathrm{K}$ y $\mathrm{Mg}$ en el tiempo (Cuadro 8). El Ca en los primeros $15 \mathrm{~cm}$ de suelo presentó un decrecimiento significativo $(\alpha=0,05)$ entre los 85 y 265 dds, al mostrar en esta última fecha mayores contenidos de $\mathrm{Ca}$, con $12 \mathrm{C}(31,6$ $\left.\mathrm{cmol}(+) .1^{-1}\right)$ y $50 \mathrm{C}\left(30,6 \mathrm{cmol}(+) . \mathrm{l}^{-1}\right)$ y difirieron estadísticamente del testigo $\left(25,6 \mathrm{cmol}(+) \cdot \mathrm{l}^{-1}\right)$. El $\mathrm{K}$ en los primeros $15 \mathrm{~cm}$ de profundidad a los 85 dds mostró la mayor cantidad en la prueba 50C $\left(2,11 \mathrm{cmol}(+) \cdot \mathrm{l}^{-1}\right)$, mientras que el testigo mostró el menor contenido $\left(0,4 \mathrm{cmol}(+) \cdot \mathrm{l}^{-1}\right)$. A los $265 \mathrm{dds}$ el $\mathrm{K}$ mostró un incremento significativo, al ser 50C, 37C y el testigo, los que mostraron mayor contenido $\left(3,7-3,6 \mathrm{cmol}(+) \cdot \mathrm{l}^{-1}\right)$. Al evaluar el $\mathrm{K} \mathrm{a}$ una profundidad de $15-30 \mathrm{~cm}$ mostró el mismo comportamiento de acumulación que las capas superficiales, sin embargo, las concentraciones son menores. Las cantidades de $\mathrm{Mg}$ incrementaron significativamente en el tiempo para las
2 profundidades, se encontró que a los 265 dds los tratamientos $37 \mathrm{C}, 50 \mathrm{C}$ y testigo mostraron diferencias a la mayor presencia de este nutrimento y que el de $12 \mathrm{C}$, con la menor cantidad. A profundidad de 15 - 30 el testigo mostró la menor dosis, sin embargo, no difiere estadísticamente de la prueba de $12 \mathrm{C}$.

Se identificó que el Si en el suelo disminuyó con el tiempo mientras, que el $\mathrm{P}$ y $\mathrm{Zn}$ aumentaron significativamente (Cuadro 9). Al evaluar la concentración de $\mathrm{Si}$ en los primeros $15 \mathrm{~cm}$ de profundidad, se encontró que el testigo presentó las concentraciones más bajas $(\alpha=0,05)$ al compararlas con los demás experimentos. Este comportamiento también se encontró a profundidades de $15-30 \mathrm{~cm}$, sin embargo, la concentración de Si en las pruebas no varió en el tiempo. El P mostró un incremento importante entre los 175 dds y 265 dds en los primeros $15 \mathrm{~cm}$ de profundidad. Se encontró que el testigo mostró la mayor concentración de $\mathrm{P}$ en las últimas 2 fechas de evaluación $\left(163,0-227,2 \mathrm{mg} . \mathrm{l}^{-1}\right)$ y difieren del resto de las prácticas realizadas $\left(121-182 \mathrm{mg} . \mathrm{l}^{-1}\right)$. 


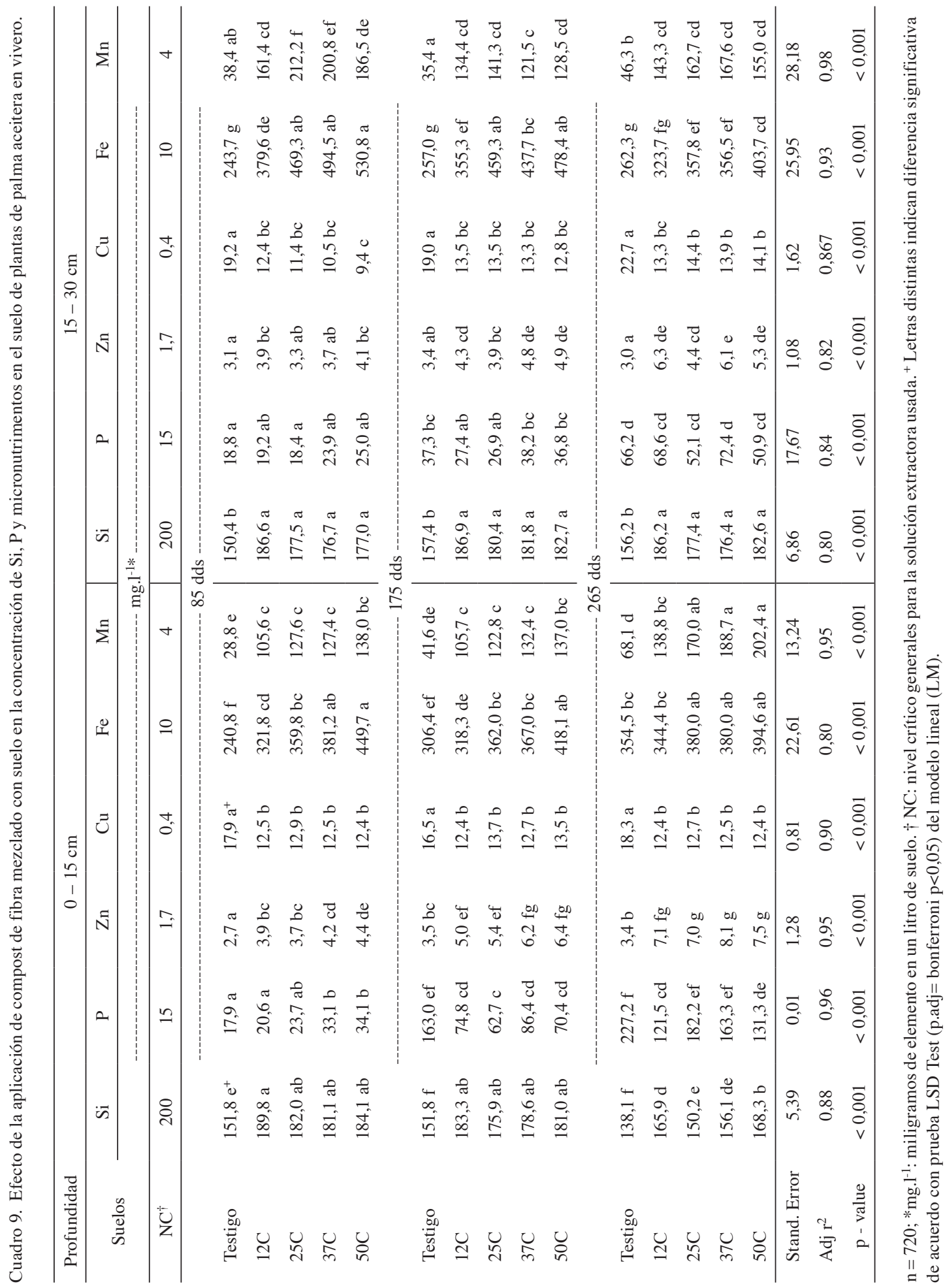


Efecto de las mezclas de compost de fibra y suelo en la absorción de nutrimentos en las plantas

La absorción de nutrimentos según el abc no mostró para los nutrimentos $\mathrm{N}, \mathrm{P}, \mathrm{K}, \mathrm{Ca}, \mathrm{Mg}$, $\mathrm{S}, \mathrm{Cu}, \mathrm{Zn}$ y B diferencias mínimas significativas $(\alpha=0,05)$ y en el testigo sobresale, que se encontraron tendencias de mayor acumulación en el de 12C (Cuadro 10). El Si mostró una mayor acumulación con $12 \mathrm{C}$ y no difiere con los $37 \mathrm{C}$ y el testigo. Al comparar la acumulación de $\mathrm{Al}$, se encontró que a $12 \mathrm{C}$ mostró la mayor absorción de este nutrimento, sin embargo, no fue diferente estadísticamente con respecto a los 37C. Esta misma tendencia de acumulación se presentó con el Fe en los mismos tratamientos, incluyendo el 25C. El Mn mostró la mayor acumulación en el testigo y presentó diferencias significativas al compararlo con el resto de los experimentos.

Cuadro 10. Índice de áreas bajo la curva en absorción de nutrimentos en plantas de palma aceitera, tratadas con compost de fibra en mezcla con suelo en vivero.

\begin{tabular}{|c|c|c|c|c|c|c|c|}
\hline \multirow{2}{*}{ Tratamientos } & $\mathrm{N}$ & $\mathrm{P}$ & $\mathrm{K}$ & $\mathrm{Ca}$ & $\mathrm{Mg}$ & $\mathrm{S}$ & $\mathrm{Si}$ \\
\hline & \multicolumn{7}{|c|}{ 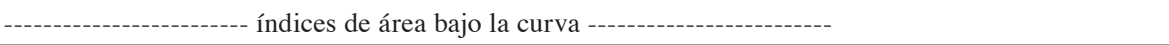 } \\
\hline Testigo & 196,6 a & 20,3 a & $153,1 \mathrm{a}$ & 92,0 a & 29,1 a & 21,9 a & $98,7 \mathrm{ab}$ \\
\hline $12 \mathrm{C}$ & 237,7 a & 23,3 a & $179,7 \mathrm{a}$ & 100,4 a & $29,5 \mathrm{a}$ & $25,0 \mathrm{a}$ & $114,0 \mathrm{a}$ \\
\hline $25 \mathrm{C}$ & 205,9 a & $20,4 \mathrm{a}$ & $154,4 \mathrm{a}$ & 82,6 a & $25,1 \mathrm{a}$ & $21,7 \mathrm{a}$ & $86,1 \mathrm{~b}$ \\
\hline $37 \mathrm{C}$ & 217,0 a & $22,4 \mathrm{a}$ & $153,8 \mathrm{a}$ & $82,8 \mathrm{a}$ & $27,0 \mathrm{a}$ & $22,6 \mathrm{a}$ & $92,1 \mathrm{ab}$ \\
\hline $50 \mathrm{C}$ & $206,0 \mathrm{a}$ & $21,0 \mathrm{a}$ & $146,6 \mathrm{a}$ & $78,6 \mathrm{a}$ & $24,9 \mathrm{a}$ & $19,8 \mathrm{a}$ & $74,3 \mathrm{~b}$ \\
\hline Stand. Error & 23,4 & 2,43 & 19,24 & 10,4 & 3,24 & 2,61 & 11,1 \\
\hline Adj r2 & 0,72 & 0,73 & 0,73 & 0,74 & 0,70 & 0,71 & 0,81 \\
\hline $\mathrm{p}$ - value & 0,012 & 0,01 & 0,02 & $<0,01$ & 0,02 & 0,02 & $<0,01$ \\
\hline \multirow{2}{*}{ Tratamientos } & $\mathrm{Al}$ & $\mathrm{Fe}$ & $\mathrm{Cu}$ & $\mathrm{Zn}$ & $\mathrm{Mn}$ & B & - \\
\hline & \multicolumn{7}{|c|}{ 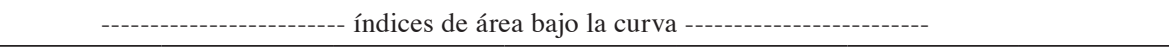 } \\
\hline Testigo & $77999,0 \mathrm{~b}$ & $7205,7 \mathrm{~b}$ & $261,7 \mathrm{a}$ & 287,9 a & 1197,4 a & 301,3 a & - \\
\hline $12 \mathrm{C}$ & 112371,6 a & 11735,6 a & $267,5 \mathrm{a}$ & 333,6 a & 867,4 b & $255,5 \mathrm{a}$ & - \\
\hline $25 \mathrm{C}$ & $70532,2 \mathrm{~b}$ & $9339,9 a b$ & $213,3 \mathrm{a}$ & 278,3 a & $838,4 \mathrm{~b}$ & $224,5 \mathrm{a}$ & - \\
\hline $37 \mathrm{C}$ & $86103,9 \mathrm{ab}$ & $9754,9 \mathrm{ab}$ & $246,6 \mathrm{a}$ & 306,7 a & 881,4 b & 237,8 a & - \\
\hline $50 \mathrm{C}$ & $65987,9 \mathrm{~b}$ & $7770,6 \mathrm{~b}$ & $235,1 \mathrm{a}$ & 259,4 a & 801,4 b & 247,7 a & - \\
\hline Stand. Error & 11490 & 1173 & 40,9 & 35,94 & 119,7 & 33,6 & - \\
\hline Adj r2 & 0,83 & 0,82 & 0,54 & 0,70 & 0,82 & 0,75 & - \\
\hline $\mathrm{p}$ - value & $<0,001$ & 0,001 & 0,13 & 0,02 & 0,001 & $<0,01$ & - \\
\hline
\end{tabular}

$\mathrm{n}=1680 ;{ }^{+}$Letras distintas indican diferencia significativa de acuerdo con prueba LSD Test $(\mathrm{p}$. adj $=$ bonferroni $\mathrm{p}<0,01) \mathrm{del}$ modelo lineal (LM).

$\mathrm{Al}$ calcular la eficiencia de fertilización de los nutrimentos $\mathrm{N}, \mathrm{P}, \mathrm{K}, \mathrm{Mg}, \mathrm{S}$ en expresión de fertilizantes $\left(\mathrm{N}, \mathrm{P}_{2} \mathrm{O}_{5}, \mathrm{~K}_{2} \mathrm{O}, \mathrm{MgO}, \mathrm{SO}_{4}\right)$ se encontró que la tendencia de mayores eficiencias las presentó el tratamiento $12 \mathrm{C}(43,9-19,0 \%)$, mientras que las eficiencias más bajas las manifestó el testigo (12,0 - 2,8\%) (Cuadro 11). El B mostró la mayor tendencia de eficiencia de fertilización en 
Cuadro 11. Comparación de eficiencia de fertilización en nutrimentos aplicados en tratamientos con distintas fuentes de silicio en mezcla al suelo en vivero de palma aceitera.

\begin{tabular}{|c|c|c|c|c|c|c|c|c|c|}
\hline Tratamiento & Rubro & unidades & $\mathrm{N}$ & $\mathrm{P}_{2} \mathrm{O}_{5}$ & $\mathrm{~K}_{2} \mathrm{O}$ & $\mathrm{MgO}$ & $\mathrm{SO}_{4}$ & B & $\mathrm{BP}$ \\
\hline \multirow{3}{*}{$12 \mathrm{C}$} & Requisito & \multirow{2}{*}{ g.planta ${ }^{-1}$} & 3,5 & 0,8 & 3,0 & 0,7 & 1,2 & 0,005 & - \\
\hline & Dosis aplicada & & 13,7 & 4,1 & 11,1 & 2,1 & 2,8 & 0,26 & - \\
\hline & eficiencia & $\%$ & 25,3 & 19,0 & 27,2 & 32,4 & 43,9 & 1,75 & 24,9 \\
\hline \multirow{3}{*}{$25 \mathrm{C}$} & Requisito & \multirow{2}{*}{ g.planta ${ }^{-1}$} & 2,7 & 0,7 & 2,9 & 0,7 & 1,0 & 0,004 & - \\
\hline & Dosis aplicada & & 13,7 & 4,1 & 11,1 & 2,1 & 2,8 & 0,26 & - \\
\hline & eficiencia & $\%$ & 19,6 & 17,3 & 25,7 & 32,4 & 38,1 & 1,63 & 22,5 \\
\hline \multirow{3}{*}{$37 \mathrm{C}$} & Requisito & \multirow{2}{*}{ g.planta ${ }^{-1}$} & 2,6 & 0,7 & 2,5 & 0,6 & 0,9 & 0,004 & - \\
\hline & Dosis aplicada & & 13,7 & 4,1 & 11,1 & 2,1 & 2,8 & 0,26 & - \\
\hline & eficiencia & $\%$ & 19,3 & 16,3 & 22,9 & 26,7 & 33,4 & 1,67 & 20,1 \\
\hline \multirow{3}{*}{$50 \mathrm{C}$} & Requisito & \multirow{2}{*}{ g.planta ${ }^{-1}$} & 3,2 & 0,7 & 2,8 & 0,6 & 1,1 & 0,005 & - \\
\hline & Dosis aplicada & & 13,7 & 4,1 & 11,1 & 2,1 & 2,8 & 0,26 & - \\
\hline & eficiencia & $\%$ & 23,3 & 16,9 & 24,9 & 28,4 & 38,5 & 2,03 & 22,3 \\
\hline \multirow{3}{*}{ Testigo } & Requisito & \multirow{2}{*}{ g.planta ${ }^{-1}$} & 2,8 & 0,6 & 2,5 & 0,6 & 1,0 & 0,005 & - \\
\hline & Dosis aplicada & & 23,0 & 23,0 & 50,0 & 14,0 & 14,0 & 0,52 & - \\
\hline & eficiencia & $\%$ & 12,0 & 2,8 & 5,1 & 4,6 & 7,4 & 0,99 & 5,5 \\
\hline
\end{tabular}

$\mathrm{n}=260 ;{ }^{+}$Letras distintas indican diferencia significativa de acuerdo con prueba LSD Test (p.adj= bonferroni $\left.\mathrm{p}<0,01\right)$ del modelo lineal $(\mathrm{LM})$. $\mathrm{BP}=$ balance porcentual (promedio de los \%).

el tratamiento 50C, mientras que el testigo mostró la menor eficiencia.

\section{Análisis multivariado de la absorción de nutrimentos en las plantas y concentración de nutrimentos en el suelo en relación con la severidad de CNF}

Al calcular el comportamiento dimensional de la variabilidad con el análisis de componentes principales (ACP), se asigna un $77,1 \%$ en las variables al comportamiento del suelo y $95,4 \%$ a las variables de acumulación de nutrimentos en las 2 primeras dimensiones (Figura 1). El análisis de agrupamiento se realizó para encontrar una relación entre la severidad del CNF en el tiempo con las variables químicas de suelo y absorción de nutrimentos en la planta. El ACP en las primeras 3 dimensiones en las variables de suelo, logró explicar un 77,1\% de la variación, ya que en la primera parte logra explicar el $46,5 \%$ y en la segunda un $30,6 \%$. En las variables de acumulación de nutrimentos, se identificó que la primera dimensión un $89,0 \%$ y en la segunda un $6,4 \%$, esto determinó que las variables mostraron poca variación y son altamente correlativas. 

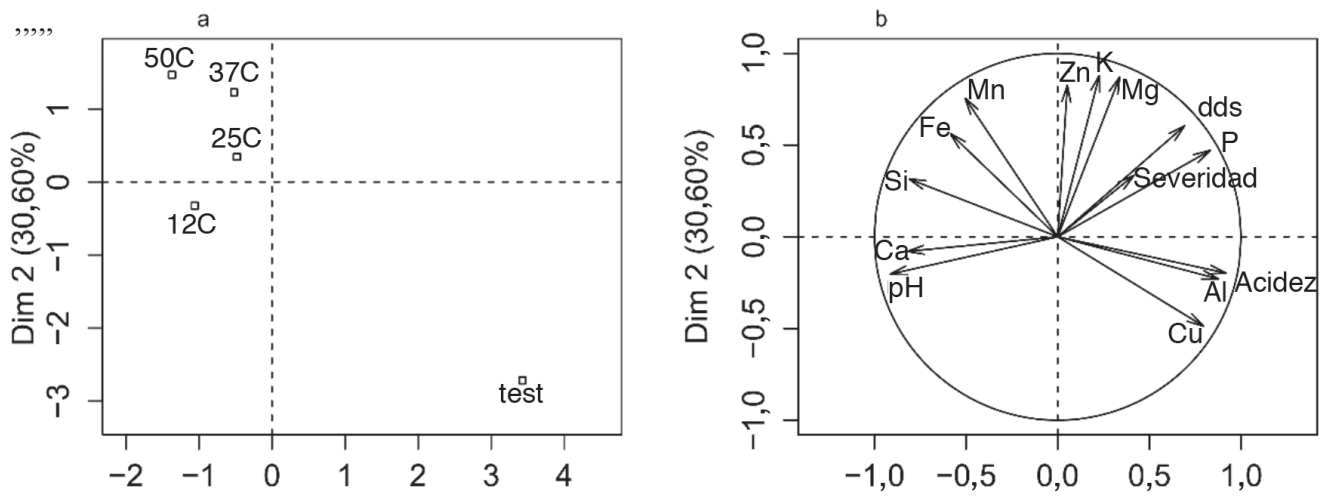

$\operatorname{Dim} 1(46,53 \%)$
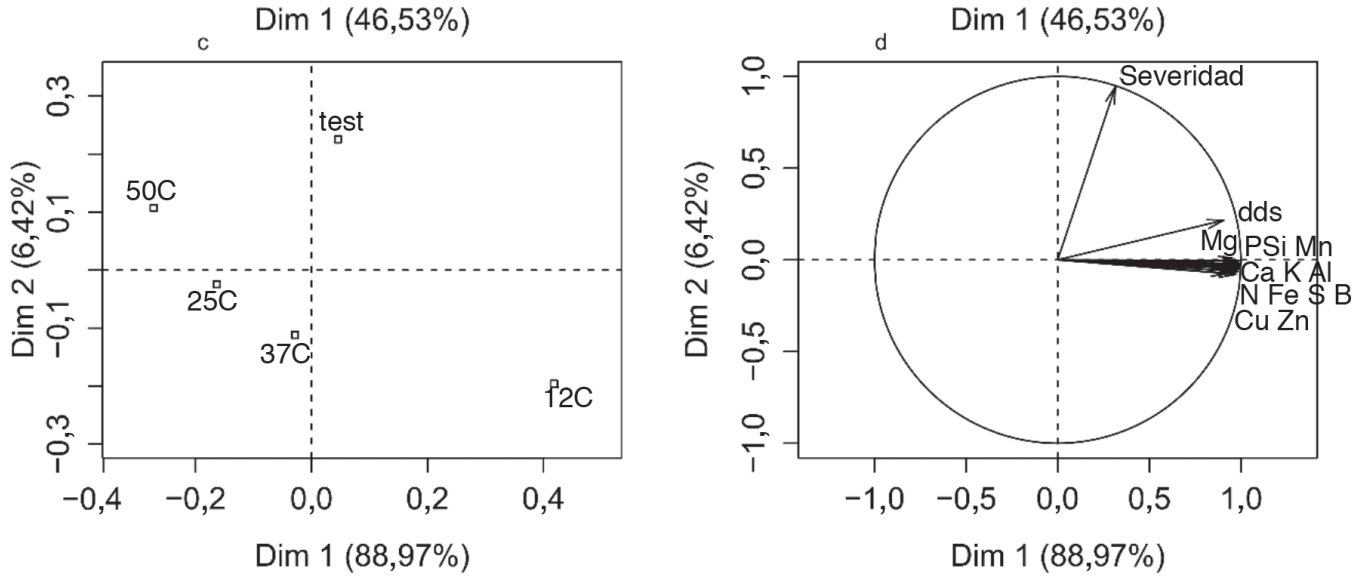

Fig. 1. Análisis de componentes principales para el contenido nutricional en el suelo, acumulación y severidad en palmas de vivero. a) Mapa de factor de individuos en dimensiones 1 y 2 para concentración de nutrientes en suelos b) mapa de factor de variables en dimensiones 1 y 2 para concentración de nutrientes en suelos. c) mapa de factor de individuos en dimensión 1 y 2 para acumulación de nutrientes en plantas. d) mapa de factor de variables en dimensiones 2 y 3 para acumulación de nutrientes en plantas.

El comportamiento dimensional de los nutrimentos en las mezclas de compost con el suelo mostró un agrupamiento importante entre las mezclas de mayor proporción de fibra (Figura 1a, b). La dimensión 1 del ACP, se apartó del testigo con mezcla de compost, mientras que la dimensión 2 se apartó con 50C, 37C y 25C respecto a $12 \mathrm{C}$ y el testigo. Lo que determinó que a $12 \mathrm{C}$ hubo un comportamiento nutricional distinto al testigo y a su vez, estos tienen comportamientos que fueron distintos a los $50 \mathrm{C}, 37 \mathrm{C}$ y $25 \mathrm{C}$. Al evaluar las correlaciones de los nutrimentos el análisis multivariado mostró que la severidad presentó una correlación directa con el tiempo (dds) $\mathrm{y}$ fuerte con los contenidos de $\mathrm{P}$, mientras que $\mathrm{K}$, Zn y Mg mostró una correlación débil. Además, el Ca y pH mostraron una correlación inversa con el tiempo y la severidad, lo cual posiblemente influiría con las enfermedades foliares.

El comportamiento de la acumulación de los nutrimentos en las plantas no mostró una relación directa con la enfermedad foliar (Figura 1c, d). Al comparar el agrupamiento dimensional de los individuos, se encontró que el tratamiento $12 \mathrm{C}$ 
presentó una relación distinta al testigo $(6,4 \%)$ y que ambos mostraron comportamientos diferentes a los demás tratamientos (89\%). Al comparar las variables en el mapa de factores, se encontró que todos los nutrimentos acumulados mostraron una relación directa con los dds y presentaron una relación débil con la severidad total en la planta.

\section{Análisis físicos de suelos}

Las mezclas de compost de fibra mejoraron significativamente los parámetros físicos de los suelos en las bolsas de vivero (Cuadro 12). Al comparar los parámetros físicos a los 90 dds, se encontró que la infiltración en las bolsas con mezclas de compost mostró diferencias $\left(42,1-92,7 \mathrm{~mm} \cdot \mathrm{min}^{-1}\right)$ altamente significativas $(<$ $\left.1 \times 10^{-4}\right)$ en comparación al testigo $\left(9,5 \mathrm{~mm} \cdot \mathrm{min}^{-1}\right)$. La densidad aparente (Dap) disminuyó conforme aumentó el porcentaje de compost en el suelo, donde el testigo mostró la mayor Dap $(0,98$ g. $\mathrm{cm}^{-3}$ ) y se diferenció significativamente de los tratamientos $25 \mathrm{C}, 37 \mathrm{C}$ y $50 \mathrm{C}$, con valores de $0,88,0,81$ y 0,75 g.cm ${ }^{-3}$ respectivamente. La conductividad hidráulica $(\mathrm{CH})$ aumentó en relación con el porcentaje de fibra en mezcla con el suelo y el testigo mostró la menor $\mathrm{CH}\left(5,20 \mathrm{~cm} \cdot \mathrm{h}^{-1}\right)$ con diferencias al resto de tratamientos.

Cuadro 12. Efecto de la aplicación de compost en las características físicas del suelo en las bolsas de vivero de palma.

\begin{tabular}{|c|c|c|c|c|c|c|}
\hline \multirow[t]{2}{*}{ Tratamientos } & $\begin{array}{l}\text { Infiltración } \\
\left(\mathrm{mm} \cdot \mathrm{min}^{-1}\right)\end{array}$ & $\begin{array}{c}\text { Dap } \\
\left(\text { g.cm }{ }^{-3}\right)\end{array}$ & $\begin{array}{c}\mathrm{CH} \\
\left(\mathrm{cm} \cdot \mathrm{h}^{-1}\right)\end{array}$ & $\begin{array}{l}\text { Infiltración } \\
\left(\mathrm{mm} \cdot \mathrm{min}^{-1}\right)\end{array}$ & $\begin{array}{c}\text { Dap } \\
\left(\text { g.cm } \mathrm{cm}^{-3}\right)\end{array}$ & $\begin{array}{c}\mathrm{CH} \\
\left(\mathrm{cm} \cdot \mathrm{h}^{-1}\right)\end{array}$ \\
\hline & \multicolumn{3}{|c|}{--------------- 90 dds --------------- } & \multicolumn{3}{|c|}{-------------- 300 dds -------------- } \\
\hline $12 \mathrm{C}$ & 42,1 & 0,92 & 15,0 & 31,3 & 0,91 & 30,2 \\
\hline $25 \mathrm{C}$ & 42,8 & 0,88 & 34,2 & 59,0 & 0,89 & 34,4 \\
\hline $37 \mathrm{C}$ & 92,7 & 0,81 & 43,4 & 40,8 & 0,80 & 55,0 \\
\hline $50 \mathrm{C}$ & 54,0 & 0,75 & 60,5 & 115,5 & 0,81 & 66,8 \\
\hline Testigo & 9,5 & 0,98 & 5,20 & 2,29 & 0,93 & 4,9 \\
\hline Comparaciones & \multicolumn{6}{|c|}{-- Dunnett test $\operatorname{Pr}(>|\mathrm{t}|)$} \\
\hline $12 \mathrm{C}-$ Testigo & $<1 \times 10^{-4}$ & 0,184 & 0,793 & $<1 \times 10^{-10}$ & 0,715 & 0,050 \\
\hline $25 \mathrm{C}-$ Testigo & $<1 \times 10^{-4}$ & 0,008 & 0,041 & $<1 \times 10^{-10}$ & 0,137 & 0,018 \\
\hline $37 \mathrm{C}$ - Testigo & $<1 \times 10^{-4}$ & $<0,001$ & 0,002 & $<1 \times 10^{-10}$ & $<0,001$ & $<0,001$ \\
\hline $50 \mathrm{C}$ - Testigo & $<0,001$ & $<0,001$ & $<0,001$ & $<1 \times 10^{-10}$ & $<0,001$ & $<0,001$ \\
\hline
\end{tabular}

$\mathrm{n}=400$; Infiltración instantánea, Dap = Gramos de partícula + espaciamiento en partículas en un volumen determinado; $\mathrm{CH}=$ Conductividad hidráulica; Probabilidades por debajo de $5 \%(\operatorname{Pr}(>|\mathrm{t}|)<0,05)$ representan diferencias significativas de tratamientos en comparación al testigo. 
Las mezclas de compost de fibra mantuvieron en el tiempo las mejoras de los parámetros físicos evaluados en los suelos de las bolsas de vivero. (Cuadro 12). Al evaluar los mismos parámetros a los 300 dds, se encontró que la infiltración mostró diferencias altamente significativas cuando se compararon los tratamientos (31,3 $\left.115,5 \mathrm{~mm} \cdot \mathrm{min}^{-1}\right)$ con el testigo $\left(2,29 \mathrm{~mm} \cdot \mathrm{min}^{-1}\right)$. En relación con la Dap los tratamientos 37C y $50 \mathrm{C}$ mostraron significativamente la mayor densidad al compararlo con el testigo. Todos los tratamientos con compost presentaron la mayor $\mathrm{CH}$ que el testigo.

\section{DISCUSIÓN}

Las plantas de palma aceitera mostraron mayor tolerancia al Complejo de Necrosis Foliar con $12 \%$ de fibra, no obstante, los demás no mostraron reducción en la severidad de CNF. El crecimiento también se presentó con mayores tendencias a los $12 \mathrm{C}$ y es probable que al tener una mayor tolerancia a las enfermedades foliares, el crecimiento en estas plantas fue mayor que las demás pruebas. Esto se observó detalladamente a los 300 dds, debido a que el diámetro de bulbo mostró un incremento altamente significativo en comparación con el testigo y mayor en relación con las demás pruebas. Los efectos concuerdan con Ramírez y Muños (2011), Ulloa y Serrano (2012), donde determinaron que CNF, o bien enfermedades foliares, mostraron reducciones importantes en el diámetro de bulbo, largo de raquis y número de hojas.

El análisis de crecimiento no mostró diferencias significativas entre los tratamientos. Es probable que las características químicas de las fibras semicomposteadas no beneficiaron en la nutrición de las plantas en vivero. Aunque posee relaciones C:N ideales > 20\% (Lozada et al. 2014), las plantas con dosis crecientes de fibra no presentaron incrementos en las fechas evaluadas. Sin embargo, se encontró que por arriba de un $25 \%$ de compost en mezcla con el suelo tuvieron reducciones con el vigor de las plantas precisamente con el diámetro de bulbo.
Probablemente por el porcentaje de potasio que aporta las fibras y la acumulación que mostraron los análisis químicos en el tiempo, tuviera atribución con la absorción de micronutrimentos de cargas positivas $\left(\mathrm{Zn}^{+}\right)$.

La absorción de Zn en las plantas de palma aceitera no mostró diferencias significativas al comparar con los tratamientos en el tiempo, sin embargo, mostró tendencias de mayor acumulación en el tratamiento $12 \mathrm{C}$. Al observar esta tendencia se analizó la de $\mathrm{Zn}$ a los 300 días y se encontró la mayor absorción significativa, en el tratamiento $12 \mathrm{C}$. El N, P, K, Ca, Mg, S y Cu también mostró una tendencia de mayor absorción, pero no fueron significativos. Esto demostró que es muy probable que la absorción de $\mathrm{Zn}$ en palmas de vivero pueda tornar más tolerantes a las enfermedades foliares. Forde (1968) mencionó que la aplicación de elementos traza, o bien, micronutrimentos en vivero de palma aceitera, no mostraron resultados precisos, debido a la alta variabilidad entre las plantas, no obstante, concuerda que un balance en las concentraciones de nutrimentos en el suelo puede propiciar un mayor crecimiento en las palmas. A nivel nutricional, Datnoff et al. (2007) determinaron que el $\mathrm{Zn}$ actúa contra los hongos estrictamente al moderar la acumulación de ácido cítrico, en forma de un compuesto quelatante, además, el $\mathrm{Zn}$ envuelve muchas funciones celulares, que induce a formas de antibióticos en la planta.

La acumulación de nutrimentos en el suelo y mezclas de sustrato producto de la fertilización probablemente sea otra causa del problema de enfermedades foliares en palma aceitera. Este parámetro se demostró al observar la acumulación de macronutrimentos en el suelo en el tiempo. La absorción de nutrimentos en las plántulas no se favoreció por el exceso de nutrimentos en el suelo. Al comparar la eficiencia de fertilización (EF) se encontró que estos no sobrepasaron $44 \%$, lo que indicó que hubo un exceso de fertilización y se debería hacer un ajuste en el programa de fertilización del vivero. Asimismo, es probable que el efecto de la mezcla $12 \mathrm{C}$ favoreciera a un balance idóneo para que la absorción de otros 
cationes ( $\mathrm{Zn}$ ) fuera balanceada en las plantas y no extrínsecamente afectado por el efecto antagónicos entre otros cationes de mayor abundancia (Ca) en el suelo.

El uso de curvas de absorción mejoró la EF en la nutrición de vivero. Esto se respalda cuando se comparó las EF del testigo con el resto de los tratamientos, donde el plan nutricional de este, se efectuó según las recomendaciones generales para vivero de palma y promovió las eficiencias más bajas según el balance porcentual. El plan nutricional con fibras se realizó mediante el uso de curvas de absorción estudiadas por Ramírez y Muñoz (2010), donde los tratamientos mostraron mayor porcentaje de EF según el balance porcentual. Esto también respaldaría que a $12 \mathrm{C}$, se presentó el mayor balance porcentual al igual que mayor tolerancia a enfermedades. Lo anterior respalda la teoría de Velasco (1999), quien mencionó que las plantas enfermas con una nutrición balanceada pueden resistir más el efecto de los patógenos, lo cual se observa en un mejor desarrollo y rendimiento de la propia planta.

Por otro lado, los parámetros físicos evaluados en los suelos de las bolsas mejoraron significativamente en todos los tratamientos con compost de fibra al compararlos con el testigo. Si bien es cierto, no se encontró una relación directa de las variables físicas con la severidad, estos parámetros presentaron un comportamiento similar en su comportamiento físico en el tiempo. El ACP encontró una relación similar entre las variables químicas del suelo. Es probable que las características fisicoquímicas de las fibras en mezcla con suelo, sean diferentes al comportamiento fisicoquímico del suelo por sí solo, que se encuentra contenido en las bolsas. El mejoramiento en las características fisicoquímicas de los suelos de las bolsas, se logró a partir de la aplicación de $12 \%$ de compost.

\section{CONCLUSIONES}

La aplicación de fibra de frutas vacías de palma aceitera mostró efectos significativos en la tolerancia de enfermedades en mezclas de $12 \%$ de compost con el suelo. Las plantas expuestas a $12 \%$ de fibra mostraron mayor vigor de plantas a los 300 dds, una tendencia de mayor acumulación de Zn y una mejor eficiencia de fertilización. Las características fisicoquímicas de los suelos mezclados con compost, mejoraron significativamente en comparación al testigo y entre las que no se encontraron diferencias importantes. Las mezclas por arriba de $25 \%$ de compost no mejoraron el crecimiento de las plantas de vivero de palma aceitera.

\section{LITERATURA CITADA}

Abdi, H; Williams, L. 2010. Principal component analysis. Wiley Interdiscip. Rev. Comput. Stat. 2(4):433-459.

ASD. 2010. Establishment and management of oil palm nurseries (en línea). Coto 47, Costa Rica. Consultado 19 abr. 2013. Disponible en http://www.asd-cr.com/ images/nurserie-guide.pdf

Cabalceta, G; Molina,E. 2006. Niveles críticos de nutrimentos en suelos de Costa Rica utilizando la solución extractora Mehlich 3. Agronomía Costarricense 30(2):31-44.

Cruz-Ruiz, E; Cruz-Ruiz, A; Aguilera-Gómez, L. 2012. Efecto en las características edáficas de un bosque templado por el cambio de uso de suelo. Tierra Latinoamericana 30(2):189-197.

Datnoff, L; Elmer, W; Huber, D. 2007. Mineral nutrition and plant disease. American Phytopathological Society Press. St Pail. USA. 278 p.

Fairhurst, T; Härdter, R. 2012. Palma aceitera. Manejo para rendimientos altos y sostenibles. International Plan Nutrition Institute (IPNI) y International Potash Institute (IPI). Ecuador. 404 p.

Ferrera, R; Alarcon, A. 2001. La microbiología del suelo en la agricultura sostenible. Ciencias Naturales y Agropecuarias 8(2):175-183.

Forde, S. 1968. The trace element nutrition of oil palm seedlings. Journal of Niger. Inst. Oil Palm Res 5(17):77-88.

Forsythe, W. 1980. Física de suelo. Manual de laboratorio. Instituto Interamericano de Ciencias Agrícolas. San José, Costa Rica. 212 p.

Henry, J. 2015. Evaluación de terapias para la recuperación de palmas aceiteras (Elaeis guineensis Jacq.) afectadas por la pudrición del cogollo (PC). Tesis Lic. Universidad de Costa Rica. San José, Costa Rica. $71 \mathrm{p}$.

Jolliffe, I. 2005. Principal Component Analysis. In Everitt, B.S., Howell, D.C. (eds.), Encyclopedia of Statistics in Behavioral Science. John Wiley \& Sons, Ltd., Chichester, UK. 488 p. 
Lobo, A. 2013. Validación de un protocolo para la determinación de silicio ( $\mathrm{Si}$ ) en muestras de suelo y tejidos vegetales. Tesis Lic. Universidad de Costa Rica. San José, Costa Rica. 95 p.

Lozada, J; Soriano, P; Costa, M. 2014. Relaciones suelo - Vegetación en una toposecuencia del Escudo Guayanés, Venezuela. Rev. Biología Tropical 62(1):385-408.

Miranda, E; Panduro, G. 2014. Determinación del tiempo de descomposición del escobajo de palma aceitera (Elaeis guinensis Jacq.), bajo condiciones ambientales en el distrito de campo verde-provincia de coronel portillo. Revista Científica Tzhoecoen 6(1):179-194.

Negreros-Castillo, P; Apodaca-Martínez, M; Mize, C. 2010. Efecto de sustrato y densidad en la calidad de plántulas de cedro, caoba y roble. Madera bosques 16(2):7-18.

Ortiz, R; Fernández, O. 2000. Cultivo de palma aceitera. EUNED, San José, Costa Rica. 208 p.

Ramírez, F; Muñoz, F. 2010. Curva de nutrientes para la etapa de vivero de tres materiales de palma aceitera (Elaeis guineensis Jacq.) (en línea). In Congreso ecuatoriano de la ciencia del suelo. Santo Domingo, Ecuador. Consultado 19 abr. 2013. Disponible en http://www. secsuelo.org/wp-content/uploads/2015/06/2.-FloriaRamirez.-Curva-materiales-vivero.-PALMATICACosta-Rica.pdf

Reyes, R; Rodríguez, H; Peña, E; Bastidas, S. 2008. Crecimiento de vivero de materiales comerciales de palma aceitera (Elaeis guinensis Jacq.) en Tumaco, Colombia. Rev. CORPOICA - Ciencia y Tecnología Agropecuaria 9(2):12-18.

Reyes, R; Rodríguez, N; Peña, E; Bastidas, S; Ibarra, N. 2009. Evaluación del crecimiento de cuatro genotipos de palma aceitera (Elaeis guineensis Jacq.) en dos tipos de suelo de diferentes características físicas. I. Vivero. Ponencia presentada In II Seminario Experiencias en la Inversión en Ciencia y Tecnología para el Sector Agropecuario Colombiano. Bogotá, Colombia.
Rodríguez, D; Ramírez, N; García, J. 2015. Evaluación de la incidencia de la producción de compost, usando biomasa de la planta de beneficio, en la huella de carbono del aceite de palma. Estudio de caso. Revista Palmas 36(1):27-39.

Rodríguez, R; Jiménez, E. 2013. Procedimiento para la evaluación de medidas de crecimiento de la hoja 1 y muestreo foliar en plantas de vivero. Boletín Mensual de la Unidad de Investigación de Palma Tica S.A. y Cukra Development Corporation. 8 p.

Serrano, R. 2003. Introducción al análisis de datos experimentales: tratamiento de datos en bioensayos. Universitat Jaume I, Castellón de la Plana, España. $189 \mathrm{p}$.

Torres, J. 2013. Caracterización morfo-fisiológica de palmas aceiteras (Elaeis guinensis Jacq.) afectadas por la pudrición del cogollo (PC) en Costa Rica. Tesis Lic. Universidad de Costa Rica. San José, Costa Rica. $73 \mathrm{p}$.

Ulloa, M; Ramírez, F. 2011. Resultado del diagnóstico sobre la necrosis foliar del vivero Coto 49. Boletín Mensual de la Unidad de Investigación de Palma Tica S.A. y Cukra Development Corporation. 3 p.

Ulloa, M; Serrano, E. 2012. Prueba de sensibilidad in vitro de Colletotrichum gloeosporioides y Curvularia lunata, principales agentes causales del Complejo de Necrosis Foliar (CNF) a diferentes fungicidas. Boletín Mensual de la Unidad de Investigación de Palma Tica S.A. y Cukra Development Corporation. 9 p.

Umaña, C; Chinchilla, C; Richardson, D. 1990. Material de desarrollo avanzado en viveros de palma aceitera (Elaeis guineensis) II. Condiciones del sustrato. Turrialba 40(1):440-451.

USDA (United States Department of Agriculture). 2001 Soil quality test kit guide. Soil Quality Institute. Washington, $88 \mathrm{p}$.

Velasco, V. 1999. Papel de la nutrición mineral en la tolerancia a las enfermedades de las plantas. Revista Terra 17(3):193-200. 